\title{
Neighbourhood characteristics and social isolation of people with psychosis: a multi-site cross-sectional study
}

\author{
Domenico Giacco ${ }^{1,2,7}$ (D) James B. Kirkbride ${ }^{3}$ Anna O. Ermakova ${ }^{2,4} \cdot$ Martin Webber $^{5} \cdot$ Penny Xanthopoulou ${ }^{6}$. \\ Stefan Priebe ${ }^{2}$
}

Received: 7 April 2021 / Accepted: 31 October 2021 / Published online: 17 November 2021

(c) The Author(s) 2021

\begin{abstract}
Purpose People with psychosis are vulnerable to social isolation, which is associated with worse clinical outcomes. In general populations, people living in areas with higher population density have more social contacts, while those living in more socially deprived and fragmented areas are less satisfied with their relationships. We assessed whether and how neighbourhood factors are associated with social contacts and satisfaction with friendships for people with psychosis.

Methods We carried out a cross-sectional study including people with psychosis aged 18-65 years in urban and rural sites in England. Population density and social deprivation and fragmentation indexes were described within Lower Level Super Output Areas (LSOA). Their associations with participants' social contacts and satisfaction with friendships were tested with negative binomial and ordinal regression models, respectively.

Results We surveyed 511 participants with psychotic disorders. They had a median of two social contacts in the previous week (interquartile range $[\mathrm{IQR}]=1-4$ ), and rated satisfaction with friendships as 5 out of 7 (Manchester Short Assessment of Quality of Life; IQR =4-6). Higher population density was associated with fewer social contacts (Z-standardised relative risk $[R R]=0.88 ; 95 \% C I=0.79-0.99, p=0.03)$, but not with satisfaction with friendships $(\mathrm{RR}=1.08 ; 95 \% \mathrm{CI}=0.93-1.26$, $p=0.31$ ). No associations were found for social contacts or satisfaction with friendships with social deprivation or fragmentation indexes.

Conclusions Clinicians in urban areas should be aware that their patients with psychosis are more socially isolated when more people live around them, and this could impact their clinical outcomes. These findings may inform housing programmes.
\end{abstract}

Keywords Social isolation $\cdot$ Population density $\cdot$ Social deprivation $\cdot$ Social fragmentation $\cdot$ Schizophrenia

Domenico Giacco

domenico.giacco@warwick.ac.uk

1 Division of Health Sciences, Warwick Medical School, University of Warwick, Gibbet Hill Campus, Coventry CV4 7AL, England

2 Unit for Social and Community Psychiatry, (WHO Collaborating Centre for Mental Health Service Development), Barts and the London School of Medicine, Queen Mary University of London, Newham Centre for Mental Health, London E13 8SP, England

3 Division of Psychiatry, University College London, London W1T 7BN, England
4 Department of Life Sciences, Faculty of Natural Sciences, Imperial College London, Silwood Park, Buckhurst Road, Ascot, Berks SL5 7PY, England

5 Department of Social Policy and Social Work, University of York, Heslington YO10 5DD, York, UK

6 College of Medicine and Health, University of Exeter, St Luke's Campus, Exeter EX2 4TH, UK

7 Present Address: Coventry and Warwickshire Partnership NHS Trust, Coventry, England 


\section{Introduction}

Social isolation is a predictor of early mortality and of poor physical and psychological health outcomes in the general population [1-3]. It is, therefore, a serious clinical concern for people with psychotic disorders who have, on average, fewer social contacts $[4,5]$ and are less satisfied with their social relationships than the general population and other people with mental or physical health conditions [6-8]. Higher levels of social isolation are linked with more severe symptoms $[6,9]$ and higher use of inpatient services [10]. Social support has been found to facilitate recovery from psychosis from patient perspective [7].

Vulnerability to social isolation can be partially explained by individual-level variables, such as more severe symptomatology [6-8, 11], unemployment or single marital status [4], which predict some of the differences in subjective (e.g. satisfaction with social relations, loneliness) and objective (e.g. social network size, number of social contacts in a specific timeframe) indicators of social isolation. However, a large amount of variation in measures of social isolation amongst people with psychosis remains unexplained.

One possibility is that the wider social environment of a person experiencing psychosis may affect their degree of social isolation as the environment is strongly linked with other aspects of psychosis. For example, areas with higher levels of social deprivation, fragmentation (i.e., the absence of connections between individuals and society), and population density have higher incidence rates of psychotic disorders [12-15]. Furthermore, the use of services by people with severe mental illness appears to be greater in areas with higher social deprivation [16, 17], while an increase in population density is linked to lower hospitalisation rates [16].

To identify whether there was any evidence of associations between neighbourhood-level characteristics and subjective and objective indicators of social isolation, we carried out a systematic appraisal of the literature (see box 1 for methodology used). We identified six crosssectional studies of general populations (non-clinical samples). Three studies [18-20] reported that the number of social contacts increased in areas with higher social propinquity (i.e., physical or psychological proximity), and one study found that people living in more densely populated areas had more social contacts, even if not necessarily with neighbours [21]. Two studies showed that higher social deprivation and fragmentation were associated with subjective aspects of social isolation, such as reduced social trust [22] and greater loneliness [23].

Our systematic search did not identify any studies assessing the associations of neighbourhood-level characteristics and social contacts of people with psychotic disorders, despite their vulnerability to social isolation $[4,6]$.

\section{Box 1: background literature search- methods}

We searched EMBASE, MEDLINE, and Web of Science for studies published in any language. The review was carried out as a background of this work and later updated to March 31, 2020. Our search terms were "neighbourhood" OR "social deprivation" OR "social fragmentation" OR "population density" AND "social contacts" OR "social isolation". We also screened references of reviews in related areas.

\section{Aims of the study}

In this study, we assessed the relationship between neighbourhood-level factors and objective (social contacts involving at least a brief conversation) and subjective (satisfaction with friendships) indicators of social isolation.

\section{Materials and methods}

\section{Study design and participants}

In the period between the beginning of June 2017 and the end of May 2018, we conducted a cross-sectional survey in community mental health teams across six participating NHS Trusts covering a range of geographical areas, in both urban and rural contexts: Cornwall Partnership NHS Foundation Trust; Devon Partnership NHS Trust; East London NHS Foundation Trust (covering East London, Luton and Bedfordshire); Oxford Health NHS Foundation Trust (covering large areas of Oxfordshire and Buckinghamshire), and Somerset Partnership NHS Foundation Trust; Tees, Esk and Wear Valleys NHS Foundation Trust (covering county Durham, Darlington, Teeside and North Yorkshire). Participants were identified from secondary mental health care service caseloads from clinicians or clinical study officers.

Participants were included if they conformed to the following conditions: were aged 18-65 years; had a clinical diagnosis of a psychotic disorder according to the International Classification of Disease-10 (ICD-10) codes F20-29, as identified in clinical records; were receiving care from outpatient secondary mental health services or primary care services; had capacity to provide informed consent; and were able to communicate in English. Participants were excluded if they had a current and primary diagnosis of substance use disorder (ICD-10, F10-19), had 
been hospitalised in the previous week (although these potential participants could be re-approached at a later time), or their postcodes could not be obtained because they were homeless or living in temporary accommodation at the time of the survey. All participants provided written informed consent.

\section{Procedures and measures}

Eligible participants were identified by members of their wider clinical team and asked for their consent to speak to a researcher. Participants then completed the study questionnaires at the presence of the researcher. Participants could either complete the questionnaire themselves or ask the researcher to read out the questions for them and complete the questionnaires, based on their verbal instructions. Researchers also obtained consent to access participant clinical records to retrieve clinical and socio-demographic characteristics. Data was entered into a database held on a secure server.

The questionnaire asked participants to self-report on two measures. First, using the Social Contacts Assessment (SCA) [24], participants reported the number of social contacts in the previous week. According to the SCA, a "social contact" was someone the participants could name and with whom they would have had at least a brief face-to-face conversation (more than just greeting) in the last week. Participants were asked not to include people they were living with or mental healthcare professionals. For employed participants, people they worked with could only be included if contacts took place outside their workplace and were not related to their work. This will be referred to as an "objective measure of social contacts" as, whilst influenced by the recall and personal appraisal of social contacts of a participant, refers to contacts which have actually happened in the previous week.

The Social Contacts Assessment (SCA) is a questionnaire aimed to count social contacts in the previous week. This was used previously in an observational study [24] and a randomised controlled trial in England [25]. Its use so far showed ease of completion by participants and sensitivity to change.

The SCA is enclosed as Appendix I in the online supplementary material.

Second, participants reported satisfaction with the quality and quantity of friendships, measured using the sixth item of the Manchester Short Assessment of Quality of Life (MANSA), i.e. 'how satisfied are you with the number and quality of your friendships' [26], which was rated on a score from 1 (very dissatisfied) to 7 (very satisfied). This will be referred to as a "subjective measure of social contacts", as it assesses a subjective appraisal of social contacts and friendships which may be somewhat independent from the frequency or recency of social contacts.

The MANSA is a widely used questionnaire to assess quality of life of people with severe mental illness throughout the world. It has been validated in both the United Kingdom [26] and elsewhere [27].

We also collected additional participant characteristics such as age, gender (male/female), marital status (single/in a relationship), country of birth (born in the United Kingdom/born in a different country), education level (tertiary or higher/lower), living situation (living alone/not living alone), accommodation (living independently/living in supported accommodation), employment (employed/not employed), receipt of welfare benefits (or not), and length of illness (calculated in number of years from the day of first contact with mental health services). These were collected from participants' assessments and checked against available data in medical records.

To collect data about the residences of participants, we used Lower Layer Super Output Areas (LSOA)—defined as a small geospatial statistical unit used in the UK Census with a minimum population of 1000 and an average of 1500 designed to improve the reporting of small area statistics in England and Wales [28]. LSOA were obtained from postcodes for participants' current address at the point of assessment. To ensure confidentiality, postcodes were not stored in our database.

Neighbourhood-level characteristics of population density, index of multiple deprivation, and social fragmentation index were derived from UK 2011 Census data [28]. Population density was defined as the number of usual residents per hectare, a metric unit of area defined as 10,000 square metres or approximately 2.47 acres. The population density score was Z-standardised. The Index of Multiple Deprivation (IMD) is the official measure of relative deprivation for small areas (neighbourhoods) in England. It draws on multiple sets of data to estimate an overall rank for deprivation across several domains (income, employment, education, health, crime, barriers to services, housing quality) We used IMD scores linked to each participant's LSOA of residence from the 2011 Indices of Deprivation [29]. The Social Fragmentation Index (SFI) aims to capture aspects of the local population that may reflect a greater collective risk of social fragmentation/lack of social cohesion. The index is built from four census variables, based on the proportion of the relevant resident population/households who were: (a) unmarried persons; (b) single-person households; (c) privately rented households; (d) living at a different address in the previous year (residential mobility). IMD and SFI were Z-standardised and summed, with higher scores indicating more social fragmentation. 


\section{Statistical analysis}

Descriptive statistics (i.e., median and interquartile range [IQR]) were reported for the number of social contacts in the previous week, score of satisfaction with friendships, neighbourhood-level variables, and the socio-demographic and clinical variables described above.

One variable (length of illness) showed a higher percentage of missing values than our a-priori threshold value (5\%). Hence, multiple imputation by chained equation was used for all missing values, using all variables included in the analysis as the basis for imputation. All values of regression analyses are presented as pooled estimates following five rounds of multiple imputation procedures.

Two separate regression models were fit, which had two different outcome variables, i.e., the number of social contacts in the previous week and the satisfaction with friendships score. We treated the number of social contacts as a count variable, modelled using negative binomial regression given the evidence of overdispersion in our data $($ mean $=2.9$, variance $=6.9$ ). Our second variable (satisfaction with friendships) was ordinal, hence we used an ordinal regression to model this data.

Modelling for both variables was exploratory and proceeded as follows. Univariable a priori association of outcome variables of regression models with neighbourhoodlevel variables and participant-level variables were tested. If an association at the level of $p<0.05$ for neighbourhoodlevel characteristics (main independent variables) and at the level of $p<0.10$ participant-level characteristics (covariates) was found in univariable models, these variables were then added to the final multivariable models.

Sensitivity analyses were carried out using only complete cases and are provided in the online appendix as supplementary material. No differences in findings compared to the primary analysis were present.

All multivariable regression models were set at a significance level of $p<0.05$. We reported relative risk estimates for the association between neighbourhood-level variables and number of social contacts, and estimates from the ordinal regression model for the change in satisfaction scores, along with their $95 \%$ confidence intervals $(95 \% \mathrm{CI})$. Analyses were carried out with the Statistical Package for the Social Sciences (SPSS), version 26.0 [30].

\section{Role of the funding source}

The funders of the study had no role in study design, data collection, data analysis, data interpretation, or writing of the report. The corresponding author had full access to all the data in the study and had final responsibility for the decision to submit for publication.

\section{Results}

Inclusion criteria were met by 511 participants who were living in 390 LSOA (Fig. 1), the median of participants per LSOA was 1 , and there were a maximum of four participants per LSOA. There were no missing cases for the number of online social contacts, the population density index, the social fragmentation index and the index of multiple deprivation.

Data on satisfaction with friendships were missing for nine participants, $1.8 \%$ of the whole sample and available for 502 participants (out of 511). For all variables but one (length of illness), missing values were less than 3\%. For the length of illness, missing values were $11.8 \%$ of the total cases. All missing values were replaced via multiple imputation techniques, as described above. The median age at recruitment was 44 years (IQR 36-52), 178 (34.8\%) participants were female, and $394(77.1 \%)$ of the participants were born in the United Kingdom (Table 1). The median number of social contacts in the previous week was 2 (IQR $=1-4)$ with a median score of satisfaction with quality and quantity of friendships of 5 out of $7, \mathrm{IQR}=4-6$ ). Median population density was 50.3 people per hectare $(\mathrm{IQR}=23.3-112.8)$ and median values for the IMD and SFI

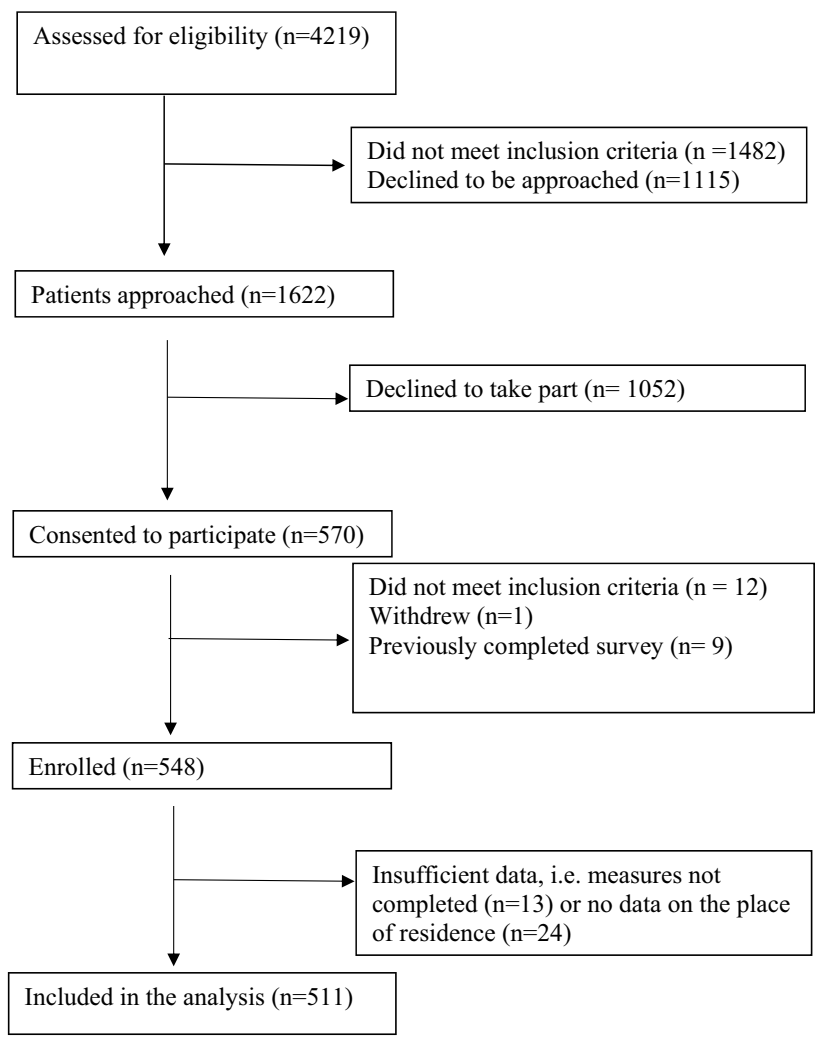

Fig. 1 CONSORT diagram 
were 29.2 (IQR $=16.8-39.8)$ and $1.8(\mathrm{IQR}=-0.3$ to -4.2$)$, respectively (Table 1).

In univariable negative binomial regression models of social contacts, people with psychosis living in areas with higher population density had fewer social contacts (relative risk $[\mathrm{RR}]=0.88,95 \% \mathrm{CI}=0.79-0.98, p=0.02$ ). No differences were found with regard to deprivation (Z-standardised $\mathrm{RR}=0.98 ; 95 \% \mathrm{CI}=0.89-1.08, p=0.73$ ) or social fragmentation (Z-standardised $\mathrm{RR}=0.98 ; 95 \%$ $\mathrm{CI}=0.88-1.08, p=0.69)$. Level of education, living alone, living in independent accommodation, being employed, and being white British were also associated $(p<0.01)$ with social contacts and hence were included in the multivariable modelling, along with population density. The length of illness did not show any association with the number of social contacts.

In multivariable models, the association between higher population density and lower number of social contacts remained after adjustment for participant-level characteristics (Z-standardised $\mathrm{RR}=0.88 ; 95 \%$ $\mathrm{CI}=0.79-0.99, p=0.03$; Table 2).

In univariable ordinal regression models of satisfaction with friendships, population density did not show a significant association with satisfaction with friendships $(\mathrm{RR}=1.08 ; 95 \% \mathrm{CI}=0.93-1.26, p=0.31)$. Social deprivation $(\mathrm{Z}$-standardised $\mathrm{RR}=1.13 ; 95 \% \mathrm{CI}=0$ 0.97-1.32, $p=0.13)$ and social fragmentation (Z-standardised $\mathrm{RR}=1.15 ; 95 \% \mathrm{CI}=0.98-1.34, p=0.08)$ also did not have significant associations with satisfaction with friendships. Hence, a multivariable model was not developed.

\section{Discussion}

We found that people with psychosis living in more densely populated areas reported fewer social contacts, in contrast with results from similar studies in general populations [18-21]. Social deprivation and social fragmentation scores were not associated with the number of social
Table 1 Socio-demographic and clinical variables

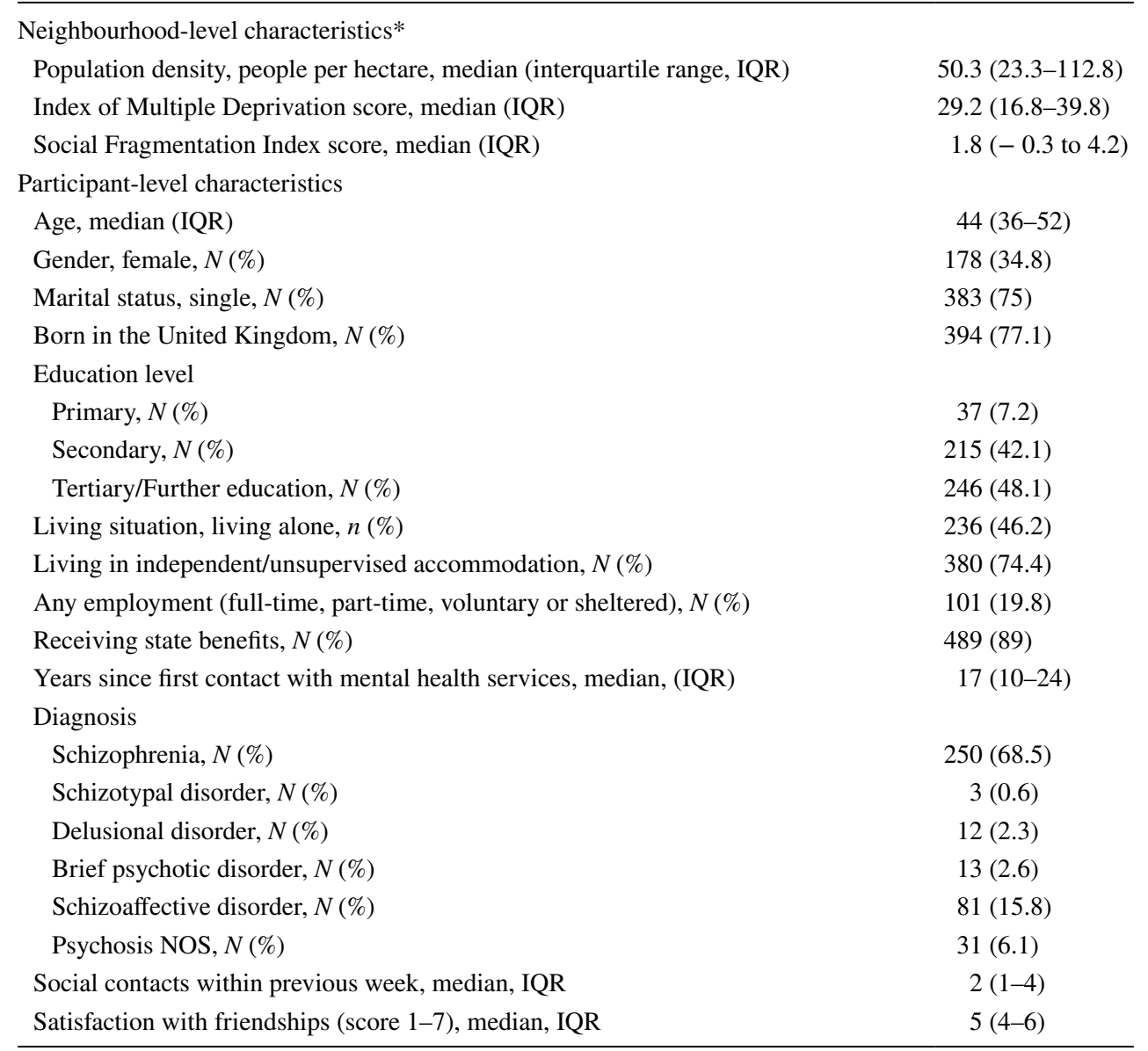

$N=511$. Data is provided on complete cases

*Original, non $Z$-standardised values 
Table 2 Univariable and multivariable negative binomial regression models testing cross-sectional associations of number of social contacts in the previous week for each participant (outcome variable) with neighbourhood-level characteristics and participant-level characteristics

\begin{tabular}{|c|c|c|c|c|c|c|}
\hline \multirow[t]{2}{*}{ Independent variables } & \multicolumn{3}{|c|}{ Univariable models } & \multicolumn{3}{|c|}{ Multivariable models } \\
\hline & $\mathrm{RR}^{*}$ & $\mathrm{CI}^{* *}(95 \%)$ & $p^{* * *}$ & $\mathrm{RR}^{*}$ & $\mathrm{CI}^{* *}(95 \%)$ & $p^{* * *}$ \\
\hline \multicolumn{7}{|l|}{ Neighbourhood-level variables $* * * *$} \\
\hline Population density & 0.88 & $0.79-0.98$ & 0.02 & 0.88 & $0.79-0.99$ & 0.03 \\
\hline Index of Multiple Deprivation & 0.98 & $0.89-1.08$ & 0.727 & & & \\
\hline Social Fragmentation Index & 0.98 & $0.89-1.08$ & 0.69 & & & \\
\hline \multicolumn{7}{|l|}{ Participant-level characteristics } \\
\hline Age (years) & 1.00 & $0.99-1.01$ & 0.39 & & & \\
\hline Gender (female versus male) & 0.85 & $0.69-1.05$ & 0.12 & & & \\
\hline Marital status (single vs not single) & 1.05 & $0.83-1.32$ & 0.69 & & & \\
\hline Tertiary or higher education (vs lower level of education) & 0.82 & $0.67-1.00$ & 0.05 & 0.87 & $0.71-1.06$ & 0.17 \\
\hline Living alone (vs. living with others) & 0.75 & $0.62-0.92$ & 0.01 & 0.76 & $0.61-0.93$ & 0.01 \\
\hline Living independently (vs. living in supervised settings) & 0.80 & $0.63-1.01$ & 0.06 & 0.90 & $0.71-1.15$ & 0.42 \\
\hline Any employment (vs. not employed) & 0.73 & $0.57-0.93$ & 0.01 & 0.76 & $0.59-0.97$ & 0.03 \\
\hline Receiving welfare benefits (vs. not receiving benefits) & 1.06 & $0.59-1.89$ & 0.85 & & & \\
\hline Years since first contact with services & 1.000 & $0.99-1.01$ & 0.93 & & & \\
\hline Born in the United Kingdom (vs. born abroad) & 0.91 & $0.71-1.16$ & 0.43 & & & \\
\hline
\end{tabular}

*Relative risk

**Confidence interval

$* * *$ Significance level set at $p<0.05$

$* * * * Z$-standardised

contacts. Subjective satisfaction with friendships was not associated with any of the considered neighbourhood-level characteristics.

This study is the first to address the question of how neighbourhood-level variables were associated with social contacts in people with psychosis. We recruited a large sample across several mental health providers covering a variety of urban and rural areas in England. We considered several potential covariates, including the length of illness, which was not associated with either number of social contacts or satisfaction with friendships, and did not confound our results. The wide spread of 511 participants across 390 areas provided a high variance in neighbourhood characteristics with no clustering effect.

Our study has some limitations. First, selection bias might have influenced our results. It is possible that people who agreed to participate had different characteristics (i.e., they had more social contacts or were more satisfied with friendships) from those who declined to participate. Their relationships with clinicians who first approached them for participation might also might have made a difference as to whether they would accept or not. Moreover, whilst we have made efforts to recruit from both secondary and primary care services, we might not have reached people with psychotic disorders who are not engaging with either of these services or have not reached the threshold for their interventions. Associations between variables tend to be more robust towards selection bias than prevalence estimates [31], but we cannot exclude that a selection bias might have also affected associations (e.g., emphasising floor or ceiling effects of the variables). Second, the number of social contacts was selfreported and could have been affected by recall or desirability bias. Third, we excluded people who were unable to communicate in English due to inability to access specific interpretation services for the study or validated versions of the measures in all the different languages which would have been required. Fourth, the cross-sectional design of our study and the inclusion of participants with prevalent diagnoses meant we were unable to determine whether the observed association between higher population density and fewer social contacts was causal. Fifth, we did not measure the number of contacts occurring within mental health services. There could have been differences in service provision (e.g. presence or absence of day care initiatives) across the different sites involved which may have influenced the amount of social support that participants will have experienced. However, we felt that if we included social contacts as part of service activities our results would have been confounded by differences in service provision and we would not be able to accurately estimate the impact of neighbourhood variables on social contacts of participants. Finally, we did not have data on how long participants had lived at their current address. Future large, longitudinal studies are required to overcome these limitations. 
As described above, our findings in a sample of people with psychotic disorders are in contrast with previous research in general populations [18-22]. Longitudinal designs are required to confirm our findings and test hypotheses as to how more densely populated environments might affect the social connections of people with psychosis. We could posit two hypotheses, which are linked to the concepts of "physical proximity" (access and opportunities for random interactions with social partners due to densely populated environments) and "psychological proximity" (sharing common interests from the outset or develops familiarity with) which were found to regulate social interactions in general populations $[18,20]$.

First, it is possible that people with psychosis are more likely to actively withdraw from social contacts in densely populated areas. Having a greater number of random social interactions may act as a stressor and exacerbate symptoms such as persecutory ideation or perceptual disturbances [32, 33]. Second, the causes for social isolation may relate to behaviours of other people towards those with psychotic disorders. Because opportunities for social interactions increase in more densely populated areas, people may become more socially selective (as they have greater choice) with whom they establish "psychological proximity" [34]. People with psychotic disorders may be viewed as less attractive social partners, especially if they have difficulty making conversation or with developing familiar relationships.

These hypotheses are not mutually exclusive. However, the lack of an association between population density in the area of residence and satisfaction with friendships might suggest a limited motivation of participants living in more densely populated areas to increase their social contacts. Moreover, our study did not identify evidence of an association between satisfaction with friendships and any of our three neighbourhood-level characteristics (population density, index of multiple deprivation, and social fragmentation index). It may be that neighbourhood-level characteristics are not as important as participant-level variables-for example, the severity of symptoms [35, 36] -in determining subjective feelings of dissatisfaction with one's own social life.

Whatever the underlying reason, reduced social contacts and small social network size are linked to early mortality and morbidity in general populations $[1,2]$ and to negative social outcomes in psychosis [4]. Therefore, the association of higher population density and fewer social contacts in people with psychosis may be of high prognostic significance for this population, even in the absence of an effect on subjective feelings of satisfaction with friendships.

Clinicians in urban areas should be aware that their patients with psychosis are even more socially isolated than those who live in less densely populated areas, despite the arguably higher number of opportunities for socialisation.

Longitudinal studies over long period of time might help to confirm these findings and identify as to whether a change of residence (e.g., from an urban to a rural area) will be followed by a change in the number of social contacts.

These studies might inform interventions to reduce social isolation of people with psychosis which are currently being developed and tested $[8,37,38]$. They could also support policy decisions on housing programmes for people with psychosis who are socially isolated and have scarce family or other social support in the area in which they usually live.

Future studies should clarify why people with psychosis have fewer social contacts in areas with higher population density. This question could be addressed in the first instance by qualitative studies and requires replication and further exploration in larger longitudinal studies. Studies should explore the attitudes and behaviour of participants and of other people living in the same neighbourhoods, and evaluate changes over time to understand how social isolation develops and/or is maintained. These studies will be an important step towards the adaptation of social interventions and rehabilitation practices to the areas in which they are delivered.

It is hoped that these studies might also help us to understand better the complex pathways and factors that lead many patients with psychotic disorders to develop and experience social isolation.

Supplementary Information The online version contains supplementary material available at https://doi.org/10.1007/s00127-021-02190-x.

Acknowledgements We would like to gratefully acknowledge the vital help and support from clinicians and clinical study officers from all the NHS Trusts involved, who helped the local promotion of this study and the identification of participants.

Author contributions DG carried out the literature review and the selection of the references. DG, AOE and SP led the study protocol development. DG, AOE, SP, MW and PX supervised data collection across the study sites. DG prepared the data for the analysis. DG did the data analysis with advice from JBK. SP and JBK supported DG in the interpretation of the results. DG takes responsibility for the integrity of the data and the accuracy of the data analysis. All authors reviewed the manuscript and contributed to its final draft.

Funding This report presents independent research funded by the National Institute for Health Research (NIHR) under the Programme Grants for Applied Research programme [RP-PG-0615-20009]. The views expressed in this publication are those of the author(s), and not necessarily those of the NIHR or the Department of Health and Social Care.

Data availability The data that support the findings of this study are available on request from the corresponding author, DG. The data are not publicly available due to the research governance requirements of protecting potentially identifiable data of participants from public 
access. Access is possible for research purposes, provided that a formal data sharing agreement is completed.

\section{Declarations}

Conflict of interest All authors declare no competing interests.

Ethical approval All procedures contributing to this work comply with the ethical standards of the relevant national and institutional committees on human experimentation and with the Helsinki Declaration of 1975 , as revised in 2008. All study materials and procedures including study protocol, case report forms, participant information materials, and informed consent forms were approved by the West MidlandsSolihull Research Ethics Committee (17/WM/0191).

Open Access This article is licensed under a Creative Commons Attribution 4.0 International License, which permits use, sharing, adaptation, distribution and reproduction in any medium or format, as long as you give appropriate credit to the original author(s) and the source, provide a link to the Creative Commons licence, and indicate if changes were made. The images or other third party material in this article are included in the article's Creative Commons licence, unless indicated otherwise in a credit line to the material. If material is not included in the article's Creative Commons licence and your intended use is not permitted by statutory regulation or exceeds the permitted use, you will need to obtain permission directly from the copyright holder. To view a copy of this licence, visit http://creativecommons.org/licenses/by/4.0/.

\section{References}

1. Pantell M, Rehkopf D, Jutte D, Syme SL, Balmes J, Adler N (2013) Social isolation: a predictor of mortality comparable to traditional clinical risk factors. Am J Public Health 103:2056-2062

2. Leigh-Hunt N, Bagguley D, Bash K, Turner V, Turnbull S, Valtorta N, Caan W (2017) An overview of systematic reviews on the public health consequences of social isolation and loneliness. Public Health 152:157-171. https://doi.org/10.1016/j.puhe.2017. 07.035

3. Wang J, Lloyd-Evans B, Giacco D, Forsyth R, Nebo C, Mann F, Johnson S (2017) Social isolation in mental health: a conceptual and methodological review. Soc Psychiatry Psychiatr Epidemiol 52:1451-1461

4. Palumbo C, Volpe U, Matanov A, Priebe S, Giacco D (2015) Social networks of patients with psychosis: a systematic review. BMC Res Notes 8:560

5. Danon L, Read JM, House TA, Vernon MC, Keeling MJ (2013) Social encounter networks: characterizing Great Britain. Proc Biol Sci 280:20131037

6. Michalska da Rocha B, Rhodes S, Vasilopoulou E, Hutton P (2018) Loneliness in psychosis: a meta-analytical review. Schizophr Bull 44:114-125

7. Temesgen WA, Chien WT, Bressington D (2019) Conceptualizations of subjective recovery from recent onset psychosis and its associated factors: a systematic review. Early Interv Psychiatry 13:181-193. https://doi.org/10.1111/eip.12698

8. Tee H, Priebe S, Santos C, Xanthopoulou P, Webber M, Giacco D (2020) Helping people with psychosis to expand their social networks: the stakeholders' views. BMC Psychiatry 20:29

9. Lamster F, Lincoln TM, Nittel CM, Rief W, Mehl S (2017) The lonely road to paranoia. A path-analytic investigation of loneliness and paranoia. Compr Psychiatry. 74:35-43. https://doi.org/10. 1016/j.comppsych.2016.12.007

10. Badcock JC, Di Prinzio P, Waterreus A, Neil AL, Morgan VA (2020) Loneliness and its association with health service utilization in people with a psychotic disorder. Schizophr Res 223:105111. https://doi.org/10.1016/j.schres.2020.05.059

11. Giacco D, McCabe R, Kallert T, Hansson L, Fiorillo A, Priebe S (2012) Friends and symptom dimensions in patients with psychosis: a pooled analysis. PLoS ONE 7:e50119

12. Kirkbride J, Barker D, Cowden F et al (2008) Psychoses, ethnicity and socio-economic status. Br J Psychiatry 193:18-24

13. Ivory VC, Collings SC, Blakely T, Dew K (2011) When does neighbourhood matter? Multilevel relationships between neighbourhood social fragmentation and mental health. Soc Sci Med $72: 12$

14. Veling W, Susser E, Selten JP, Hoek HW (2015) Social disorganization of neighborhoods and incidence of psychotic disorders: a 7-year first-contact incidence study. Psychol Med 45:1789-1798

15. O’Donoghue B, Roche E, Lane A (2016) Neighbourhood level social deprivation and the risk of psychotic disorders: a systematic review. Soc Psychiatry Psychiatr Epidemiol 51:941-950

16. Losert C, Schmauß M, Becker T, Kilian R (2012) Area characteristics and admission rates of people with schizophrenia and affective disorders in a German rural catchment area. Epidemiol Psychiatr Sci 21:371-379

17. Donisi V, Tedeschi F, Percudani M et al (2013) Prediction of community mental health service utilization by individual and ecological level socio-economic factors. Psychiatry Res 209:691-698

18. Latané B, Liu JH, Nowak A, Bonevento M, Zheng L (1995) Distance matters: physical space and social impact. Personal Soc Psychol Bull 21:795-805

19. Preciado P, Snijders TAB, Burk WJ, Stattin H, Kerr M (2012) Does proximity matter? Distance dependence of adolescent friendships. Soc Netw 34:18-31

20. Small ML, Adler L (2019) The role of space in the formation of social ties. Annu Rev Sociol 45:111-132

21. Hawley ZB (2012) Does urban density promote social interaction? Evidence from instrumental variable estimation. Rev Reg Stud 42:223-248

22. Wang Z, Zhang F, Wu F (2017) Social trust between rural migrants and urban locals in China: exploring the effects of residential diversity and neighbourhood deprivation. Popul Space Place 23:e2008. https://doi.org/10.1002/psp.2008

23. Kearns A, Whitley E, Tannahill C, Ellaway A (2015) Loneliness, social relations and health and well-being in deprived communities. Psychol Health Med 20:332-344

24. Giacco D, Palumbo C, Strappelli N, Catapano F, Priebe S (2016) Social contacts and loneliness in people with psychotic and mood disorders. Compr Psychiatry 66:59-66

25. Priebe S, Chevalier A, Hamborg T, Golden E, King M, Pistrang $\mathrm{N}$ (2020) Effectiveness of a volunteer befriending programme for patients with schizophrenia: Randomised controlled trial. Br J Psychiatry 217:477-483

26 Priebe S, Huxley P, Knight S, Evans S (1999) Application and results of the Manchester Short Assessment of Quality of Life (MANSA). Int J Soc Psychiatry 45:7-12

27 Björkman T, Svensson B (2005) Quality of life in people with severe mental illness. Reliability and validity of the Manchester Short Assessment of Quality of Life (MANSA). Nord J Psychiatry 59(4):302-306. https://doi.org/10.1080/08039480500213733

28. Office for National Statistics (2011) Census aggregate data. UK Data Service, June 2016 Edn. https://doi.org/10.5257/census/ aggregate-2011-1

29. Ministry of Housing, Communities \& Local Government (2010) Official statistics: English indices of deprivation 2010. https:// 
www.gov.uk/government/statistics/english-indices-of-depri vation-2010

30. IBM Corp (2019) IBM SPSS Statistics for Windows, Version 25.0. Armonk, NY: IBM Corp

31. Etter JF, Perneger TV (2000) Snowball sampling by mail: application to a survey of smokers in the general population. Int J Epidemiol 29:43-48

32. Howes OD, Murray RM (2014) Schizophrenia: an integrated sociodevelopmental-cognitive model. Lancet 383:1677-1687

33. Lim MH, Gleeson JF (2014) Social connectedness across the psychosis spectrum: current issues and future directions for interventions in loneliness. Front Psychiatry 5:154

34 Wahba J, Zenou Y (2005) Density, social networks and job search methods: theory and application to Egypt. J Dev Econ 78:443-473

35. Matthews T, Danese A, Wertz J, Odgers CL, Ambler A, Moffitt TE, Arseneault L (2016) Social isolation, loneliness and depression in young adulthood: a behavioural genetic analysis. Soc Psychiatry Psychiatr Epidemiol 51:339-348

36. Ludwig KA, Nye LN, Simmons GL, Jarskog LF, Pinkham AE, Harvey PD (2020) Penn DL Correlates of loneliness among persons with psychotic disorders. Soc Psychiatry Psychiatr Epidemiol 55:549-559

37. Website for the SCENE programme (2017) Funded by the National Institute for Health Research. https://scene.elft.nhs.uk/ Outputs/Intervention-Manual. Retrieved on 15 Aug 2021

38. Ma R, Mann F, Wang J, Lloyd-Evans B, Terhune J, Al-Shihabi A, Johnson S (2020) The effectiveness of interventions for reducing subjective and objective social isolation among people with mental health problems: a systematic review. Soc Psychiatry Psychiatr Epidemiol 55(7):839-876. https://doi.org/10.1007/ s00127-019-01800-z 